\title{
A New Solution Method for the Finite-Horizon Discrete-Time EOQ Problem
}

\author{
Chung-Lun Li \\ Department of Logistics \\ The Hong Kong Polytechnic University \\ Hung Hom, Kowloon, Hong Kong \\ Phone: +852-2766-7410 \\ Email: lgtclli@polyu.edu.hk
}

\section{SHORT COMMUNICATION}

November 2007

Revised April 2008 


\begin{abstract}
We consider the finite-horizon discrete-time economic order quantity problem. Kovalev and Ng (2008) have developed a solution approach for solving this problem. Their approach requires a search for the optimal number of orders, which takes $O(\log n)$ time. In this note, we present a modified solution method which can determine the optimal solution without the need of such a search.
\end{abstract}

Keywords: Combinatorial optimization; EOQ; inventory management 


\section{Introduction}

We consider the following discrete version of the finite-horizon Economic Order Quantity (EOQ) problem: There is a finite planning horizon with discrete time periods $1,2, \ldots, n$. Demand occurs at a constant rate of $\lambda>0$ units per period. Orders can be placed at the beginning of any period. When an order is placed, replenishment takes place instantaneously before the demand of that period is filled. A fixed ordering cost $c>0$ is incurred when an order is placed, and an inventory holding $\operatorname{cost} h>0$ is incurred if a unit of the item is carried from one period to the next. The initial inventory at the beginning of period 1 is zero. We would like to determine the order quantity in each time period so that the total ordering and holding cost is minimized. We denote this problem as $P$.

Problem $P$ differs from the classical EOQ problem in that the planning horizon is finite and demand occurs in discrete time periods. Problem $P$ is also a special case of the classical dynamic lot size problem, which has time-dependent demand and cost parameters. Kovalev and Ng [1] have developed an algorithm for problem $P$. Their algorithm requires a search for the optimal number of orders in the planning horizon which takes $O(\log n)$ time. In this note, we present a modified solution method which does not require such a search. Note that problem $P$ is defined slightly differently from that of Kovalev and $\mathrm{Ng}$ [1], who have assumed that a holding cost $h$ is incurred if a demand unit is satisfied by an order received in the same time period. However, this difference is very minor, because the objective function of Kovalev and Ng's model differs from that of problem $P$ by only a constant amount of $h \lambda n$.

By the zero-inventory property of the classical dynamic lot size problem (see, e.g., [4], page 82), it suffices to consider the case in which orders are placed and received only when the inventory level reaches zero. Thus, the size of each order must be a multiple of $\lambda$. Let $k$ denote the number of orders placed during the planning horizon. Let $s_{i}=\lambda x_{i}$ denote the size of the $i$ th order, where $x_{i}$ is a positive integer $(i=1,2, \ldots, k)$. The total quantity ordered, $\sum_{i=1}^{k} s_{i}$, is equal to $\lambda n$, which implies that $\sum_{i=1}^{k} x_{i}=n$. Since the $i$ th order is used to satisfy the demand of the current period as well as the demand of the next $x_{i}-1$ periods, the holding cost of the items in this order equals 
$h \lambda\left[\left(x_{i}-1\right)+\left(x_{i}-2\right)+\cdots+1\right]=\frac{h \lambda}{2} x_{i}\left(x_{i}-1\right)$. Hence, in problem $P$, the total ordering cost is $c k$, and the total inventory holding cost is $\frac{h \lambda}{2} \sum_{i=1}^{k} x_{i}\left(x_{i}-1\right)$. Since $\frac{h \lambda}{2} \sum_{i=1}^{k} x_{i}=\frac{h \lambda n}{2}$, which is a constant, problem $P$ reduces to the following discrete optimization problem:

$$
\begin{array}{ll}
\text { minimize } & c k+\frac{h \lambda}{2} \sum_{i=1}^{k} x_{i}^{2} \\
\text { subject to } & \sum_{i=1}^{k} x_{i}=n \\
& k=1,2, \ldots, n \\
& x_{i}=1,2, \ldots \quad \text { for } i=1,2, \ldots, k,
\end{array}
$$

where $k, x_{1}, x_{2}, \ldots, x_{k}$ are decision variables. We denote this problem as $P^{\prime}$. (Note: $P^{\prime}$ is the same as problem $D$ in $[1]$.

Kovalev and Ng's [1] solution method can be described as follows: First, they have shown that if $k$ is given, then the optimal decision is to place $r$ orders of size $\lambda\left(\left\lceil\frac{n}{k}\right\rceil-1\right)$ and $k-r$ orders of size $\lambda\left\lceil\frac{n}{k}\right\rceil$, where $r=k\left\lceil\frac{n}{k}\right\rceil-n$. (The sequence of these $k$ orders has no impact on the total cost.) This implies that the objective function of problem $P^{\prime}$ can be rewritten as

$$
f(k)=c k+\frac{h \lambda}{2}\left\{r\left(\left\lceil\frac{n}{k}\right\rceil-1\right)^{2}+(k-r)\left\lceil\frac{n}{k}\right\rceil^{2}\right\}
$$

or equivalently,

$$
f(k)=c k+\frac{h \lambda}{2}\left\{(2 n+k)\left\lceil\frac{n}{k}\right\rceil-k\left\lceil\frac{n}{k}\right\rceil^{2}-n\right\} .
$$

They have also shown that function $f$ is convex over the integer domain $\{1,2, \ldots, n\}$. They then propose a "three-section search" procedure for determining $k \in\{1,2, \ldots, n\}$ which minimizes $f$.

\section{Determining the Number of Orders}

To present our new solution method, we first define

$$
g(y)=(2 n+y)\left\lceil\frac{n}{y}\right\rceil-y\left\lceil\frac{n}{y}\right\rceil^{2}
$$


for $y \in(0,+\infty)$. Consider any nonnegative integer $\alpha$. For simplicity, we denote $\frac{n}{\alpha}=+\infty$ if $\alpha=0$. When $\frac{n}{\alpha+1} \leq y<\frac{n}{\alpha}$, we have $\alpha<\frac{n}{y} \leq \alpha+1$, which implies that

$$
g(y)=(2 n+y)(\alpha+1)-y(\alpha+1)^{2}
$$

or equivalently,

$$
g(y)=-\alpha(\alpha+1) y+2 n(\alpha+1)
$$

Hence, function $g$ is linear within the interval $\left[\frac{n}{\alpha+1}, \frac{n}{\alpha}\right)$. In addition, for $\alpha=1,2, \ldots$,

$$
\lim _{y \rightarrow(n / \alpha)^{-}} g(y)=\left(2 n+\frac{n}{\alpha}\right)(\alpha+1)-\frac{n}{\alpha} \cdot(\alpha+1)^{2}=\left(2 n+\frac{n}{\alpha}\right) \alpha-\frac{n}{\alpha} \cdot \alpha^{2}=g\left(\frac{n}{\alpha}\right) .
$$

Therefore, $g$ is a continuous piecewise linear function.

Kovalev and $\mathrm{Ng}$ [1] have shown that function $g$ is convex when the domain of the function is restricted to the integer set $\{1,2, \ldots, n\}$. We can extend this convexity property to the domain $(0,+\infty)$. Note that equation (2) implies that the slope of $g(y)$ is $-\alpha(\alpha+1)$ when $\frac{n}{\alpha+1} \leq y<\frac{n}{\alpha}$ for $\alpha=0,1,2, \ldots$. Hence, the slope of $g(y)$ decreases as $\alpha$ increases, which implies that the slope of $g(y)$ is nondecreasing as $y$ increases. This in turn implies that $g$ is convex over the interval $(0,+\infty)$.

Recall that the objective function of problem $P^{\prime}$ is $f(k)$ as defined in (1). We now extend the definition of function $f$ to cover the domain $(0,+\infty)$. Thus,

$$
f(y)=c y+\frac{h \lambda}{2}\left\{(2 n+y)\left\lceil\frac{n}{y}\right\rceil-y\left\lceil\frac{n}{y}\right\rceil^{2}-n\right\}=c y+\frac{h \lambda}{2}[g(y)-n]
$$

for $y \in(0,+\infty)$. Because $g$ is a continuous piecewise linear convex function, $f$ is also a continuous piecewise linear convex function. The slope of $f(y)$ equals $c-\frac{h \lambda}{2} \alpha(\alpha+1)$ when $\frac{n}{\alpha+1} \leq y<\frac{n}{\alpha}$ for $\alpha=0,1,2, \ldots$. Function $f$ attains its minimum when its slope changes from negative to nonnegative as $\alpha$ decreases. This occurs when $\alpha$ changes from $\left\lfloor\alpha^{*}\right\rfloor+1$ to $\left\lfloor\alpha^{*}\right\rfloor$, where $\alpha^{*}$ satisfies the condition

$$
c-\frac{h \lambda}{2} \alpha^{*}\left(\alpha^{*}+1\right)=0
$$

or equivalently,

$$
\alpha^{*}=\sqrt{\frac{1}{4}+\frac{2 c}{h \lambda}}-\frac{1}{2}
$$


Hence, $f(y)$ attains its minimum when $y^{*}=\frac{n}{\left[\alpha^{*}\right]+1}$. By convexity of $f$, the optimal number of orders must be either $\left\lfloor y^{*}\right\rfloor$ or $\left\lceil y^{*}\right\rceil$. Therefore, we set the number of orders to $\left\lfloor y^{*}\right\rfloor$ if $f\left(\left\lfloor y^{*}\right\rfloor\right) \leq f\left(\left\lceil y^{*}\right\rceil\right)$, and $\left\lceil y^{*}\right\rceil$ otherwise.

We demonstrate our solution method using the following example: $n=10, c=75, h=0.2$, and $\lambda=100$. In this example,

$$
\begin{aligned}
f(y) & =75 y+10[g(y)-10] \\
& =75 y+10(20+y)\left\lceil\frac{10}{y}\right\rceil-10 y\left\lceil\frac{10}{y}\right\rceil^{2}-100 .
\end{aligned}
$$

Function $f$ is depicted in Figure 1. We have $\alpha^{*}=2.28$. Thus, $f$ attains its minimum when $\alpha$ changes from 3 to 2 (i.e., when the slope of the curve changes from $c-\frac{h \lambda}{2}(3)(3+1)=-45$ to $\left.c-\frac{h \lambda}{2}(2)(2+1)=15\right)$. Hence, $y^{*}=\frac{n}{\left\lfloor\alpha^{*}\right\rfloor+1}=3.33$. Note that $f\left(\left\lfloor y^{*}\right\rfloor\right)=f(3)=565$ and $f\left(\left\lceil y^{*}\right\rceil\right)=f(4)=560$. Therefore, the optimal number of orders is $k^{*}=4$. Note also that $r=k^{*}\left\lceil\frac{n}{k^{*}}\right\rceil-n=2$. This implies that the optimal decision is to place two orders of size 200 and two orders of size 300 in the planning horizon.

\section{Concluding Remarks}

We have presented a new method for determining the optimal number of orders for the finitehorizon discrete-time EOQ model. This provides us with a simple way to determine the optimal ordering policy for the problem without using three-section search or any binary search method. However, our method can only generate an approximate solution unless we can obtain an exact value of $\alpha^{*}$ in the square root calculation in (3).

In fact, we can generate an exact solution to problem $P$ without the need of an exact value of $\alpha^{*}$. To do so, we modify our solution method as follows: We first compare the values of $\frac{1}{4}+\frac{2 c}{h \lambda}$ and $\left(n+\frac{1}{2}\right)^{2}$. If $\frac{1}{4}+\frac{2 c}{h \lambda} \geq\left(n+\frac{1}{2}\right)^{2}$, then $\alpha^{*} \geq n$. In such a case, we have $y^{*}<1$, which implies that

the optimal number of orders is equal to 1 . If $\frac{1}{4}+\frac{2 c}{h \lambda}<\left(n+\frac{1}{2}\right)^{2}$, then we obtain an approximate value $\hat{\alpha}$ of $\alpha^{*}$ with $\left|\hat{\alpha}-\alpha^{*}\right| \leq 1$, and the optimal number of orders must be equal to one of the following values: $\left\lfloor\frac{n}{\lfloor\hat{\alpha}\rfloor}\right\rfloor,\left\lfloor\frac{n}{\lfloor\hat{\alpha}\rfloor+1}\right\rfloor,\left\lfloor\frac{n}{[\hat{\alpha}\rfloor+2}\right\rfloor,\left\lceil\frac{n}{\lfloor\hat{\alpha}\rfloor}\right\rceil,\left\lceil\frac{n}{[\hat{\alpha}\rfloor+1}\right\rceil$, and $\left\lceil\frac{n}{[\hat{\alpha}\rfloor+2}\right\rceil$. Hence, we choose the best 
among these six values. (If $\lfloor\hat{\alpha}\rfloor=0$, then we choose the best one among $\left\lfloor\frac{n}{\lfloor\hat{\alpha}\rfloor+1}\right\rfloor,\left\lfloor\frac{n}{\lfloor\hat{\alpha}\rfloor+2}\right\rfloor,\left\lceil\frac{n}{\lfloor\hat{\alpha}\rfloor+1}\right\rceil$, and $\left\lceil\frac{n}{[\hat{\alpha}\rfloor+2}\right\rceil$.)

Note that this method requires only simple arithmetic operations, the floor and ceiling operations (i.e., $\lfloor\cdot\rfloor$ and $\lceil\cdot 7$ ), and the determination of $\hat{\alpha}$. Kovalev and Ng's [1] method has a running time of $O(\log n)$, assuming that the floor and ceiling operations require constant time (i.e., assuming that either the floor or the ceiling operation is in the repertoire). Here, we make the same assumption on the computational requirement on the floor and ceiling operations. Then, the computational complexity of our modified solution method is dominated by the determination of $\hat{\alpha}$. Note that $\sqrt{B}$ can be computed to accuracy $\epsilon$ in $O(\log \log (B / \epsilon))$ time for any $B>1$ (see [2,3]). This implies that $\hat{\alpha}$ can be determined in $O\left(\log \log \left(\frac{1}{4}+\frac{2 c}{h \lambda}\right)\right) \leq O\left(\log \log \left(n^{2}\right)\right)=O(\log \log n)$ time. Therefore, our modified solution method can generate an exact solution to problem $P$ in $O(\log \log n)$ time.

\section{Acknowledgments}

The author would like to thank three anonymous referees for their helpful comments and suggestions. This research was supported in part by The Hong Kong Polytechnic University under grant number 1-BBZL.

\section{References}

[1] Kovalev, A., Ng, C.T., A discrete EOQ problem is solvable in $O(\log n)$ time, European Journal of Operational Research 189 (2008) 914-919.

[2] Renegar, J., On the worst-case arithmetic complexity of approximating zeros of polynomials, Journal of Complexity 3 (1987) 90-113.

[3] Ye, Y., Combining binary search and Newton's method to compute real roots for a class of real functions, Journal of Complexity 10 (1994) 271-280.

[4] Zipkin, P.H., Foundations of Inventory Management, McGraw-Hill, Boston, 2000. 


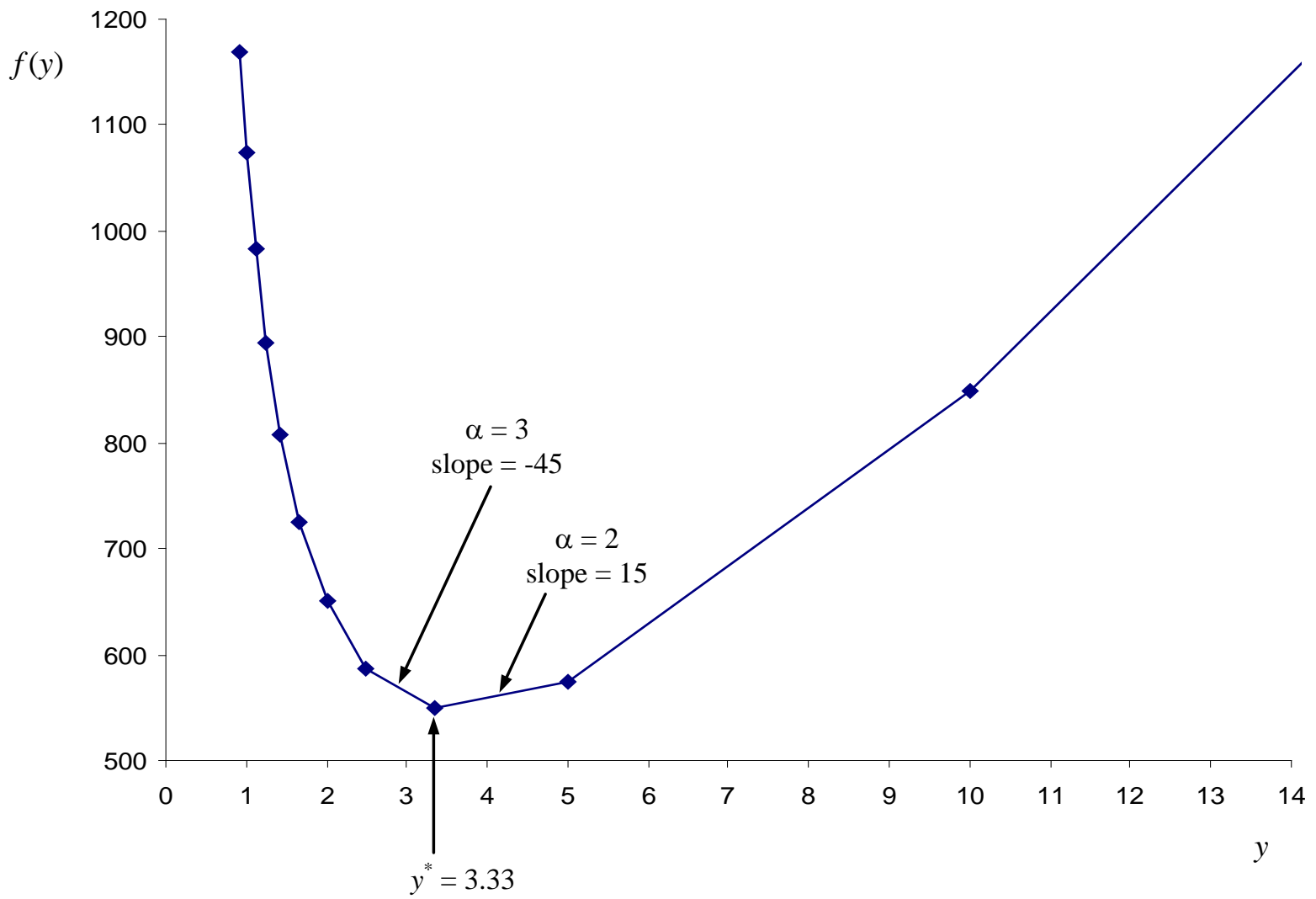

Figure 1. Function $f$ and its minimum 